\title{
Computer-Assisted Assessment of Children Drawing Movements
}

\author{
Li Fang ${ }^{12+}$, Zhao $\mathrm{He}^{2}$, Ren Tingting ${ }^{1}$ and Wu Zhongcheng ${ }^{1}$ \\ ${ }^{1}$ Institute of Intelligent Machines, Chinese Academy of Sciences, He Fei, 230031, China \\ ${ }^{2}$ Department of Automation, University of Science and Technology of China, He Fei, 230026, China
}

\begin{abstract}
The study used a computer-assisted assessment of children's drawings to investigate the relationship between schooling and fine motor skill development. 62 children from grade 1-6 performed specific drawing tasks (horizontal line, vertical line, square and circle) on a digital tablet. Six features, namely drawing time, velocity, vertical force, horizontal force, impulse of vertical force, impulse of horizontal force were extracted from the dynamic data of drawing. Further analysis showed that there was grade effect for some of these features. The results suggest dynamic data analysis could be a valuable tool to reveal the performance characteristic of fine motor ability development.
\end{abstract}

Keywords: quantitative measures, drawing movements, three-dimensional force.

\section{Introduction}

Handwriting is an essential fine motor skill that involves complicated functions. The development of handwriting begins with scribbling at an early age [1]. The ability to copy geometric forms is seen as an indication of writing readiness in the young child [2]. As the child develops, handwriting becomes more smooth and consistent.

Visual Motor Integration (VMI) has been operationally defined as the ability to copy geometric shapes [3], and the Beery Developmental Test of Visual Motor Integration (the Beery VMI test) is often used for the assessment of children's VMI skills [4]. Previous studies mainly focus on the analysis of the drawing product of participants who are required to copy figures with a pen/pencil on paper. Weil and Amundson [5] found that the kindergarten children who were able to copy the first nine VMI forms can copy more letters than the others. Marr and Cermak [6] concluded that VMI scores were significantly associated with handwriting for females. It is claimed that VMI is a useful predictor of handwriting performance in a group of first graders [7]. However, in those studies the information obtained was not comprehensive enough, in that the dynamic information in the drawing process was neglected. Furthermore, it is noticeable that the assessment was lacking in quantitative analysis, and subjective judgement and experience of researchers were highly relied on.

The aim of the present study was to exploit a computerized evaluation method of the drawing movement development using dynamic data. We selected four typical VMI forms (horizontal line, vertical line, square and circle) as templates, and collected the drawing data with a digital tablet.

\section{Method}

\subsection{Participants}

62 children from grade 1-6 were randomly recruited from a primary school. All children are right-handed, same mother-tongue and free from organic pathologies. The assent was obtained from the children and their parents.

\footnotetext{
+ Corresponding author. Tel.: + 86 18955114393; fax: +86 055165591149

E-mail address: lif@hmfl.ac.cn.
} 
Table 1. Distribution of number and age of participants

\begin{tabular}{lllllll}
\hline & Grade 1 & Grade 2 & Grade 3 & Grade 4 & Grade 5 \\
\hline Number & 10 & 8 & 10 & 13 & 11 & 10 \\
Age(years) & $6.20 \pm 0.42$ & $7.87 \pm 0.99$ & $9.00 \pm 0.47$ & $9.84 \pm 0.80$ & $11.80 \pm 0.98$ \\
\hline
\end{tabular}

\subsection{Instruments}

The children were required to execute the drawing task on a tablet using a standard shaped inkless pen. The drawing was restricted to a $7 \mathrm{~cm} \times 7 \mathrm{~cm}$ square area. During the drawing process, the trajectory position (x-coordinate $\mathrm{x}_{i}$ and $\mathrm{y}$-coordinate $\left.\mathrm{y}_{i}\right)$ and the three-dimensional pen-plane contact force $\left(F x_{i}, F y_{i}\right.$ and $\left.F z_{i}\right)$ information were acquired by the tablet with a fixed sampling rate.

Table 2. Performance parameters of the tablet

\begin{tabular}{lll}
\hline Name & Unit & Value \\
\hline Voltage & $\mathrm{V}$ & 5 \\
Power & $\mathrm{mW}$ & 300 \\
Sampling Rate & $\mathrm{Hz}$ & 100 \\
Size of Writing Area & $\mathrm{cm}^{2}$ & $7 \times 7$ \\
Position Resolution & $\mathrm{cm}$ & 0.01 \\
Force Range & $\mathrm{N}$ & $0 \sim 5$ \\
Force Resolution & $\mathrm{N}$ & 0.1 \\
\hline
\end{tabular}

\subsection{Tasks}

Considering the different education levels of the participants, we selected four simple figures. The subject was required to execute figure copying task of drawing horizontal lines, vertical lines, squares and circles. Recognized as strokes, horizontal line and vertical line are basic components of drawing trajectory. Square task is to assess the ability of orientation control, and circle task is associated with curve drawing movement.

\subsection{Procedures}

The children performed the tasks in an environment that was similar to their normal conditions at school. Before the experiment, the subject exercised for several minutes until he/she got used to drawing on the tablet. Each task was executed at least ten times. The drawing displayed on-line on the screen of a computer which was linked with the tablet.

\subsection{Data Analysis}

The drawing data is represented as:

$$
A=\left[\begin{array}{cccccc}
\mathrm{x}_{1} & \mathrm{x}_{2} & \ldots & \mathrm{x}_{\mathrm{i}} & \ldots & \mathrm{x}_{\mathrm{n}} \\
\mathrm{y}_{1} & \mathrm{y}_{2} & \ldots & \mathrm{y}_{\mathrm{i}} & \ldots & \mathrm{y}_{\mathrm{n}} \\
\mathrm{Fx}_{1} & \mathrm{Fx}_{2} & \ldots & \mathrm{Fx}_{\mathrm{i}} & \ldots & \mathrm{Fx}_{\mathrm{n}} \\
\mathrm{Fy}_{1} & \mathrm{Fy}_{2} & \ldots & \mathrm{Fy}_{\mathrm{i}} & \ldots & \mathrm{Fy}_{\mathrm{n}} \\
\mathrm{F}_{\mathrm{Z}_{1}} & \mathrm{~F}_{\mathrm{Z}_{2}} & \ldots & \mathrm{F}_{\mathrm{Zi}} & \ldots & \mathrm{F}_{\mathrm{Zn}_{\mathrm{n}}}
\end{array}\right],
$$

where $\mathrm{n}$ represents the sampling number, $\mathrm{x}_{i}, \mathrm{y}_{i}$, and $\mathrm{F} x_{i}, F y_{i}, F z_{i}$, are the $\mathrm{x}$-coordinate, y-coordinate, force in $\mathrm{X}$-axis, force in $\mathrm{Y}$-axis and force in $\mathrm{Z}$-axis at time i respectively.

For the drawing data of each subject, six features were extracted. Then the mean feature values of the drawing by the children in the same grade were calculated. The features were

- Drawing time: $\mathrm{T}=(n-1) / r$

- Velocity: $\left.V=\sum_{i=1}^{n-1}\left(y_{i+1}-y_{i}\right)^{2}+\left(x_{i+1}-x_{i}\right)^{2}\right)^{0.5} / T$

- Vertical force: $F z=\sum_{i=1}^{n} F z_{i} / n$

- Horizontal force: $F x y=\sum_{i=1}^{n}\left(F x_{i}^{2}+F y_{i}^{2}\right)^{0.5} / n$

- Impulse of vertical force: $P_{v}=\sum_{i=1}^{n} F z_{i} / p$ 
- Impulse of horizontal force: $P_{h}=\sum_{i=1}^{n}\left(F x_{i}^{2}+F y_{i}{ }^{2}\right)^{0.5} / r$

\section{Results}

One-way ANOVA was carried out to recognize the features that better highlight the differences across grades. The p-values of ANOVA were shown in Table 3. The behavior along the grades of each features is reported in Fig. 1.
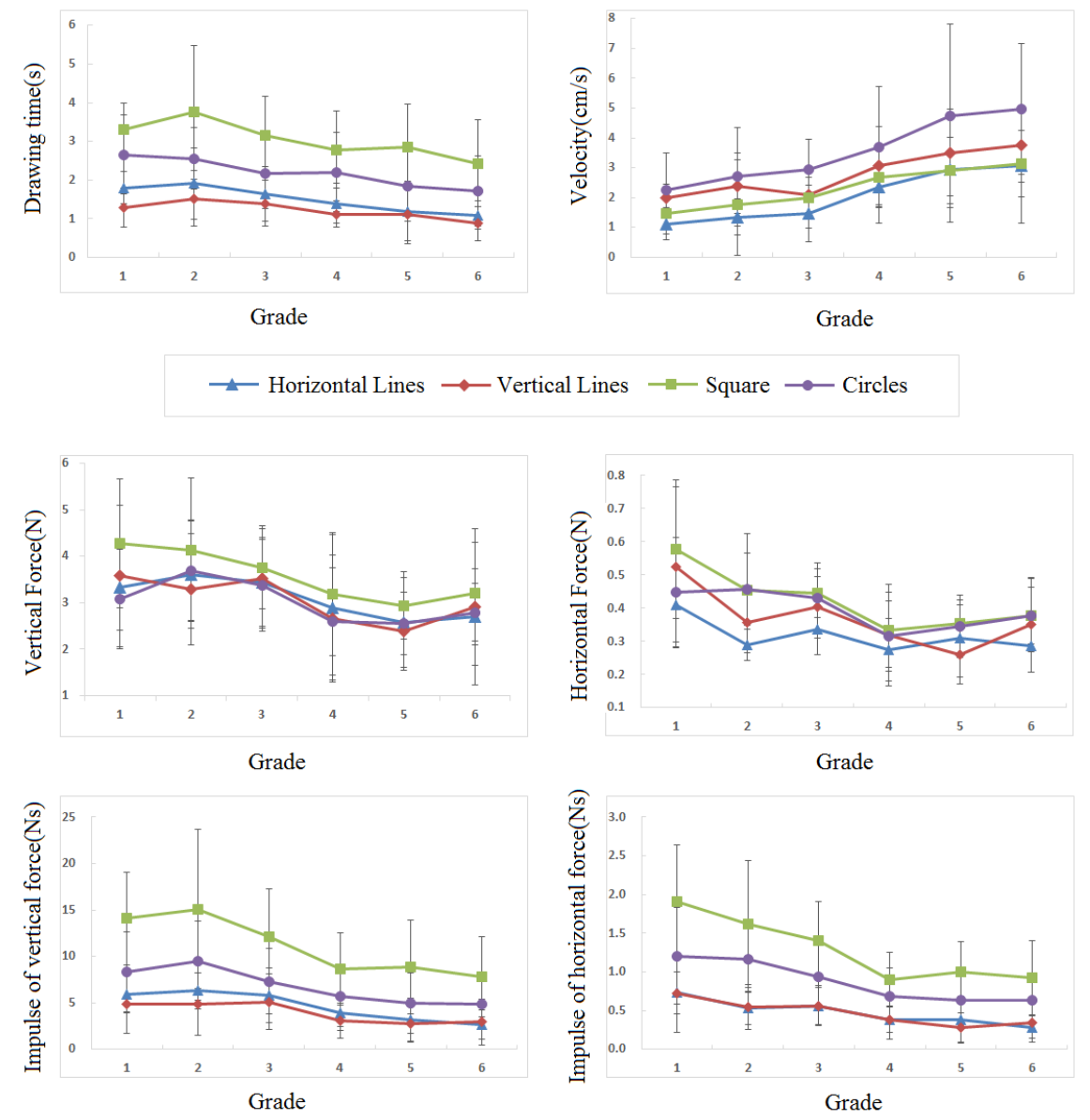

Fig. 1: Influence of grade on features.

Velocity increases significantly with grade in horizontal line drawing task $(\mathrm{p}=0.003)$, square task $(\mathrm{p}=0$. $005)$ and circle task $(\mathrm{p}=0.041)$. Participants performed greatest velocity to execute circle task.

For drawing time, vertical force, horizontal force, impulse of vertical force and impulse of horizontal force, the features value fluctuated at a few grades. However, lower value of those features in grade 4-6 compared to grade 1-3 were shown. The value of features for square task was higher than that of others.

Significant effect of grade was found at horizontal force and impulse of horizontal force for four tasks. In contrast with horizontal force, vertical force has no significant grade effect.

Table 3. Features identified by analysis of variance (ANOVA)

\begin{tabular}{llll}
\hline Feature & p-value & Feature & p-value \\
\hline Task: horizontal line & & Task: vertical line & \\
Drawing time & $0.038^{*}$ & Drawing time & 0.196 \\
Velocity & $0.003^{* *}$ & Velocity & 0.095 \\
Vertical Force & 0.259 & Vertical Force & 0.228 \\
Horizontal Force & $0.017^{*}$ & Horizontal Force & $0.004^{* *}$ \\
Impulse of vertical force & $0.001^{* *}$ & Impulse of vertical force & 0.11 \\
Impulse of horizontal & $0.001^{* *}$ & Impulse of horizontal & $0.017^{*}$ \\
force & & force &
\end{tabular}




\begin{tabular}{llll} 
Task: square & \multicolumn{3}{c}{ Task: circle } \\
Drawing time & 0.187 & Drawing time & 0.191 \\
Velocity & $0.005^{* *}$ & Velocity & $0.041^{*}$ \\
Vertical Force & 0.065 & Vertical Force & 0.067 \\
Horizontal Force & $0.001^{* *}$ & Horizontal Force & $0.029^{*}$ \\
Impulse of vertical force & $0.011^{*}$ & Impulse of vertical force & $0.006^{* *}$ \\
Impulse of horizontal & $<0.001^{* *}$ & Impulse of horizontal & $0.006^{* *}$ \\
force & & force & \\
\hline
\end{tabular}

Note: $* p<.05, * * p<.01$

\section{Discussions}

The study used a digital tablet to quantitatively measure the drawing movement of children. In the four tasks, children were required to draw on a square area with their preferred movement magnitudes and speed. Six features were extracted from dynamic drawing data. The relationships between features and schooling found in the study reflect there is an association between drawing skill and motor maturation.

In this study, the drawing duration do not have a well-defined trend, but the lower value in the higher grades was observed. It is reasonable to assume that the children spent less time on drawing tasks when their movements become increasingly efficient. The results are compatible with the finding about the duration analysis of handwriting in Italian children [8].

Velocity was found to be an important indicator of drawing ability development. The experiment result about velocity is similar to previous studies. Schooling has influence over velocity development [9], and increased velocity across grade presents the proficiency and automation of motor skill [10].

Pen pressure variation was suggested to be an effective measure on handwriting difficulties. P.I. Khalid et al. [11] concluded there is no significant difference in mean pressure between poor writers and good writers, but pen pressure of poor writers is more variable than that of good writers. Unlike the studies which only focused on vertical force of the pen-plane contact force, we also acquired horizontal force and analyzed its trend. Our finding reflects horizontal force and impulse of horizontal force had significant grade effect for all tasks, while vertical force and impulse of vertical force did not. Although the source of the drawing characteristics is the pressure applied on the pen [12], there is no consensus on the development of pen pressure up to now [10]. More attention on the research of force variability should be directed to, since force control is an essential component of fine motor movement.

\section{Acknowledgments}

This paper is supported by the National Natural Science Foundation of China (Grant No. 61273323).

\section{References}

[1] Oliver CE. A sensorimotor program for improving writing readiness skills in elementary-age children. Am $J$ Occup Ther. 1990, 44: 111-124.

[2] Katya P Feder, Annette Majnemer. Handwriting development, competency, and intervention. Developmental Medicine \& Child Neurology. 2007, 49(4): 312-317.

[3] Volman, M. J. M., Van Schendel, et al. Handwriting difficulties in primary school children: A search for underlying mechanisms. American Journal of Occupational Therapy. 2006, 60: 451-460.

[4] Beery, K. E. The VMI developmental test of visual motor integration. Administration, scoring and teaching manual. Cleveland: Modern Curriculum Press, 1989.

[5] Weil MJ, Amundson SJ. Relationship between visuomotor and handwriting skills of children in kindergarten. Am J Occup Ther. 1994, 48: 982-988.

[6] Marr D, Cermak S. Predicting handwriting performance of early elementary students with the Developmental Test of Visual-Motor Integration. Percept Mot Skills. 2002, 95: 661-669.

[7] Schneck CM. Comparison of pencil-grip patterns in first graders with good and poor writing skills. Am J Occup Ther. 1991, 45: 701-706. 
[8] Accardo, A., Genna, M., \& Borean, M. Analysis of Handwriting Kinematic Parameters in Italian Children. In V Latin American Congress on Biomedical Engineering CLAIB. Habana: Springer Berlin Heidelberg. 2011, pp. 1114-1117.

[9] Agostino P. Accardo, Mariangela Genna, et al. Development, maturation and learning influence on handwriting kinematics. Human Movement Science. 2013, 32: 136-146.

[10] Qiushi Lin, Jianfei Luo, et al. Characterization of fine motor development: Dynamic analysis of children's drawing movements. Human Movement Science. 2015, 40: 163-175.

[11] Khalid, P. I., Yunus, J., et al. Extraction of dynamic features from hand drawn data for the identification of children with handwriting difficulty. Research in Developmental Disabilities. 2010, 31(1): 256-262.

[12] Hsu, H. M., Lin, Y. C., et al. Quantification of handwriting performance: Development of a force acquisition pen for measuring hand-grip and pen tip forces. Measurement, 2013, 46(1), 506-513. 